US Army Corps of Engineers ${ }_{\circledast}$

Engineer Research and

Development Center

\title{
WERDC
}

Flood and Coastal Systems R\&D

\section{Automated Characterization of Ridge-Swale Patterns Along the Mississippi River}

Alicia D. Downard, Stephen N. Semmens, and

April 2021

Bryant A. Robbins

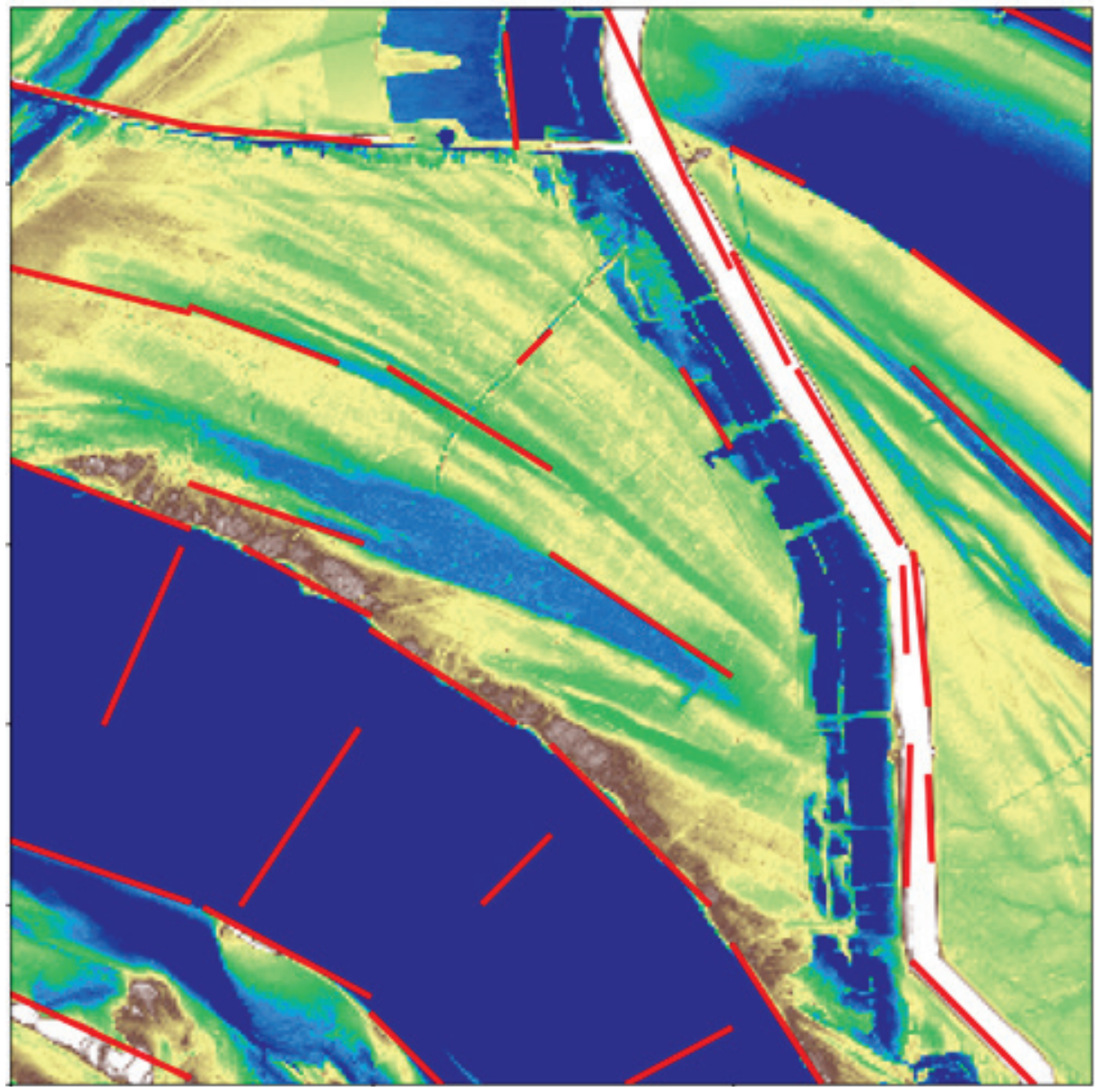


The U.S. Army Engineer Research and Development Center (ERDC) solves the nation's toughest engineering and environmental challenges. ERDC develops innovative solutions in civil and military engineering, geospatial sciences, water resources, and environmental sciences for the Army, the Department of Defense, civilian agencies, and our nation's public good. Find out more at www.erdc.usace.army.mil.

To search for other technical reports published by ERDC, visit the ERDC online library at https://erdclibrary.on.worldcat.org/discovery. 


\title{
Automated Characterization of Ridge-Swale Patterns Along the Mississippi River
}

\author{
Stephen N. Semmens and Bryant A. Robbins \\ Geotechnical and Structures Laboratory \\ U.S. Army Engineer Research and Development Center \\ 3909 Halls Ferry Road \\ Vicksburg, MS 39180 \\ Alicia D. Downard \\ Department of Geophysics, Undergraduate Student \\ Colorado School of Mines \\ 1500 Illinois Street \\ Golden, CO 80401
}

Final report

Approved for public release; distribution is unlimited.

Prepared for U.S. Army Corps of Engineers

Washington, DC 20314-1000

Under Flood \& Coastal Civil Works Direct Program, Remote Monitoring \& Sensing; Critical Infrastructure Protection and Resilience Program, Office of Homeland Security 


\section{Abstract}

The orientation of constructed levee embankments relative to alluvial swales is a useful measure for identifying regions susceptible to backward erosion piping (BEP). This research was conducted to create an automated, efficient process to classify patterns and orientations of swales within the Lower Mississippi Valley (LMV) to support levee risk assessments. Two machine learning algorithms are used to train the classification models: a convolutional neural network and a U-net. The resulting workflow can identify linear topographic features but is unable to reliably differentiate swales from other features, such as the levee structure and riverbanks. Further tuning of training data or manual identification of regions of interest could yield significantly better results. The workflow also provides an orientation to each linear feature to support subsequent analyses of position relative to levee alignments. While the individual models fall short of immediate applicability, the procedure provides a feasible, automated scheme to assist in swale classification and characterization within mature alluvial valley systems similar to LMV.

DISCLAIMER: The contents of this report are not to be used for advertising, publication, or promotional purposes. Citation of trade names does not constitute an official endorsement or approval of the use of such commercial products. All product names and trademarks cited are the property of their respective owners. The findings of this report are not to be construed as an official Department of the Army position unless so designated by other authorized documents. 


\section{Contents}

Abstract............................................................................................................................... ii

Figures...............................................................................................................iv

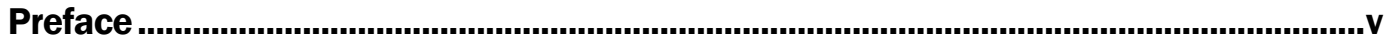

1 Introduction .......................................................................................................... 1

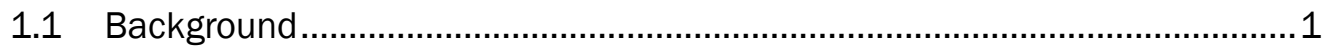

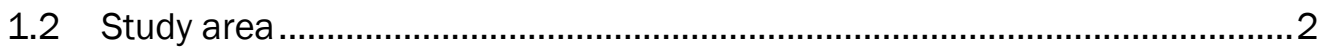

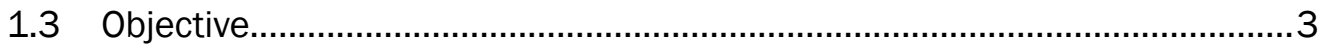

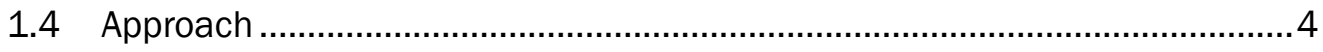

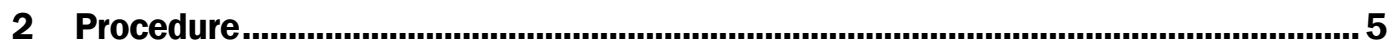

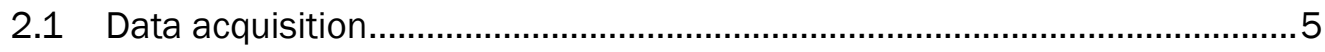

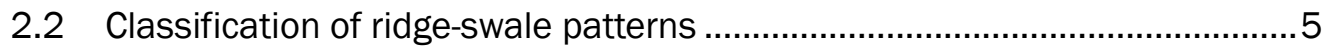

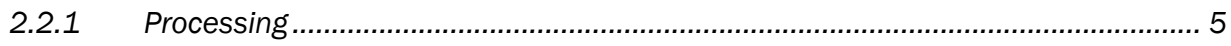

2.2.2 Classification using a convolutional neural network ............................................. 6

2.2.3 Classification using a U-net .............................................................................. 8

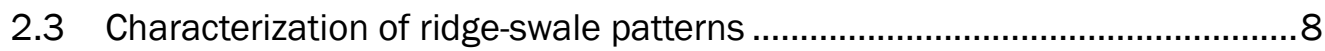

2.3.1 Highlighting linear features .............................................................................. 9

2.3.2 Application of the Hough transform ............................................................ 11

3 Results and Discussion ..........................................................................................13

3.1 Results............................................................................................. 13

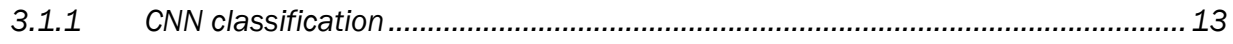

3.1.2 U-net classification..................................................................................... 13

3.1.3 Ridge-swale orientations ........................................................................ 15

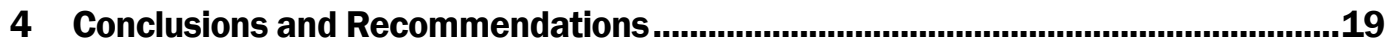

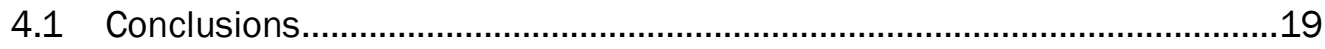

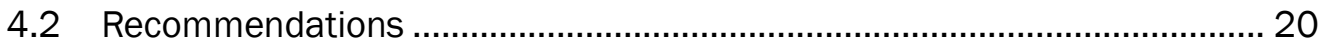

References.................................................................................................................................21

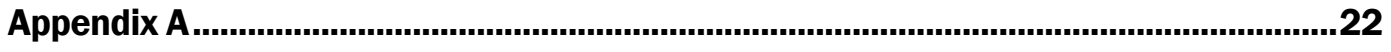

Unit Conversion Factors.....................................................................................37

\section{Report Documentation Page}




\section{Figures}

\section{Figures}

Figure 1. A ridge-swale pattern along the Mississippi River identified on a digital elevation map..

Figure 2. Aerial image of study area with reference to regional position...............................3

Figure 3. DEM of study area (Figure 2) containing interpreted swales (red lines). ............. 6

Figure 4. A depiction of the interpolation of interpreted swales (left) represented as red lines versus corresponding discretized grid values (right) represented as individual pixels shown in gray.

Figure 5. DEM (left) and associated masks after swale interpretation (middle) and Gaussian filter with thresholding (right)

Figure 6. A depiction of the spatial domain processing steps implemented on the DEM data set.

Figure 7. An example of the Hough transform applied to a filtered section of data containing a ridge-swale pattern.

Figure 8. Three different prediction results from the model trained with a U-net with the original DEM (left column), swale masks (middle column), and prediction results from the trained model (right column).

Figure 9. DEM where the characterization workflow has been applied to subsections to plot the dominant trend lines (red).

Figure 10. DEM and cross section with intersecting dominant linear trends. 


\section{Preface}

This study was conducted for the Critical Infrastructure Protection and Resilience Program, Office of Homeland Security, U.S. Army Corps of Engineers, under the direction of the Program Manager, Ms. Yazmin SedaSanabria, and the Flood and Coastal Systems R\&D Program under the direction of Dr. Julie Rosati.

The work was performed by the Geotechnical Engineering and Geosciences Branch (GS-G) of the Geosciences Division (GS), U.S. Army Engineer Research and Development Center, Geotechnical and Structures Laboratory (ERDC-GSL). At the time of publication, Mr. Christopher Price was Chief, GSG; Mr. James L. Davis was Chief, GS; and Dr. Michael K. Sharp, GST, was the Technical Director for Water Resources Infrastructure. The Deputy Director of ERDC-GSL was Mr. Charles W. Ertle II, and the Director was Mr. Bartley P. Durst.

The authors wish to acknowledge Dr. Joseph B. Dunbar of ERDC-GSL for both project assistance and inspiration. Dr. Dunbar originally conceived the idea of performing automated characterization of geomorphic features pertinent to sand boil formation from LiDAR (Light Detection and Ranging) as part of research projects on remote sensing. The authors, all working on various aspects of the problem presented, came together through a series of serendipitous events to produce the results presented.

COL Teresa A. Schlosser was the Commander of ERDC, and Dr. David W. Pittman was the Director. 


\section{Introduction}

\subsection{Background}

The Mississippi River and its tributaries form a drainage basin over $3,224,000 \mathrm{~km}^{2}$ in size in the center of the continental United States (EPA 2016). Since the Flood Control Act of 1928, the U.S. Army Corps of Engineers (USACE) has maintained comprehensive flood control and navigation infrastructure along the river, including the construction and maintenance of levees for containing flood flows (USACE 2004).

Within the Lower Mississippi Valley (LMV), levee systems sit atop a heterogeneous assortment of materials deposited by the dynamic interaction of the river with the surrounding landscape. At the ground surface, material and depositional properties may manifest in distinct topographic expressions. One such pattern, referred to as ridge-swale topography, is common throughout the alluvial valley as curvilinear, approximately parallel undulations in the topography (Lobeck 1939). Ridges represent linear regions of higher elevation, while the depressions between ridges are called swales. An example of this pattern is shown in Figure 1.

Figure 1. A ridge-swale pattern along the Mississippi River identified on a digital elevation map.
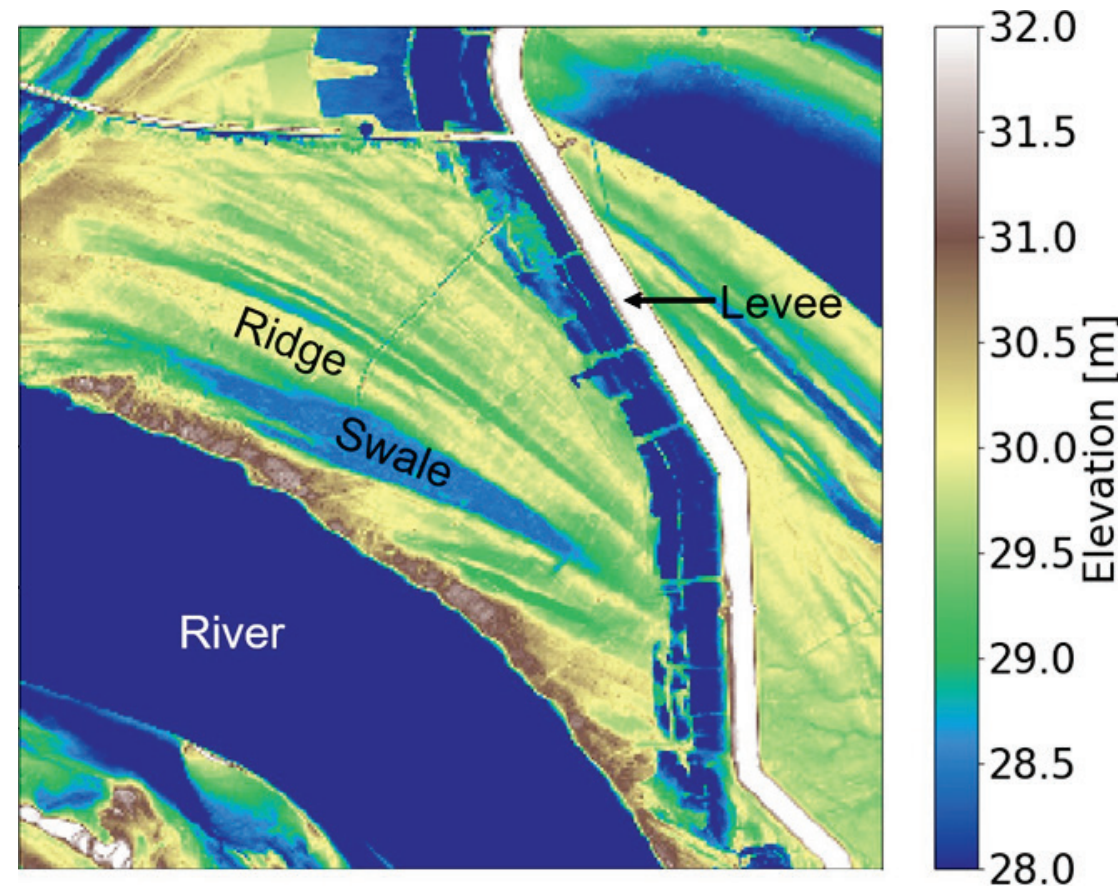
Orientations of geologic features, including swales, relative to levee structures have been shown to contribute to the likelihood of sand boils during flood events (Mansur and Kaufman 1957; Kolb 1975). Sand boils are surficial deposits of foundation sand eroded and deposited by seepage from the levee foundation. Sand boils may lead to the occurrence of backward erosion piping (BEP), wherein shallow erosion channels propagate upstream through the levee foundation (Bonelli 2013), which is a primary failure mechanism for levee embankments. Left unattended, the erosion may continue to remove foundation material until the levee embankment can no longer be supported by the foundation and failure occurs. As early as 1956, USACE observed elevated occurrences of sand boils in certain geometries of swales (Mansur and Kaufman 1957). Multiple statistical studies indicate that deposits of channel fill in addition to swales likely contribute to the spatial distribution of sand boils and thus, BEP (Glynn et al. 2012; Semmens et al. 2017).

The influence of geology on BEP can likely be explained by the interaction between water and the physical properties of materials within the respective deposit. Swales and channel fills are characteristically composed of finergrained material. Relative to the coarser material in surrounding deposits, swales and channel fill are known to focus, funnel, or otherwise significantly affect the flow of groundwater (Mansur and Kaufman 1957; Kolb 1975; Glynn et al. 2012). These deposits often contain more cohesive clay material, which better resists the erosional forces driving BEP.

Given the apparent influence of swales on the spatial occurrence of BEP, creating a process to automate the identification of swale features and map their trends could provide an essential step toward efficient characterization of BEP hazard along levee systems. The following report provides a review of research performed toward the construction of such a process.

\subsection{Study area}

The study area consists of a $2,000 \mathrm{~km}^{2}$ portion of LMV straddling the Mississippi River immediately south of the Arkansas border. A map of this region is shown in Figure 2. The region was selected for three primary reasons. First, it contains a significant number of swales for model training and assessment. Second, key data, such as digital elevation models (DEMs) and aerial imagery were readily available for the entire site. Third, the site contained a variety of other features (e.g., levees, oxbow lakes, the 
Mississippi River, and farm plots), which provided additional topographic responses to challenge and assess the final model.

Figure 2. Aerial image of study area with reference to regional position.
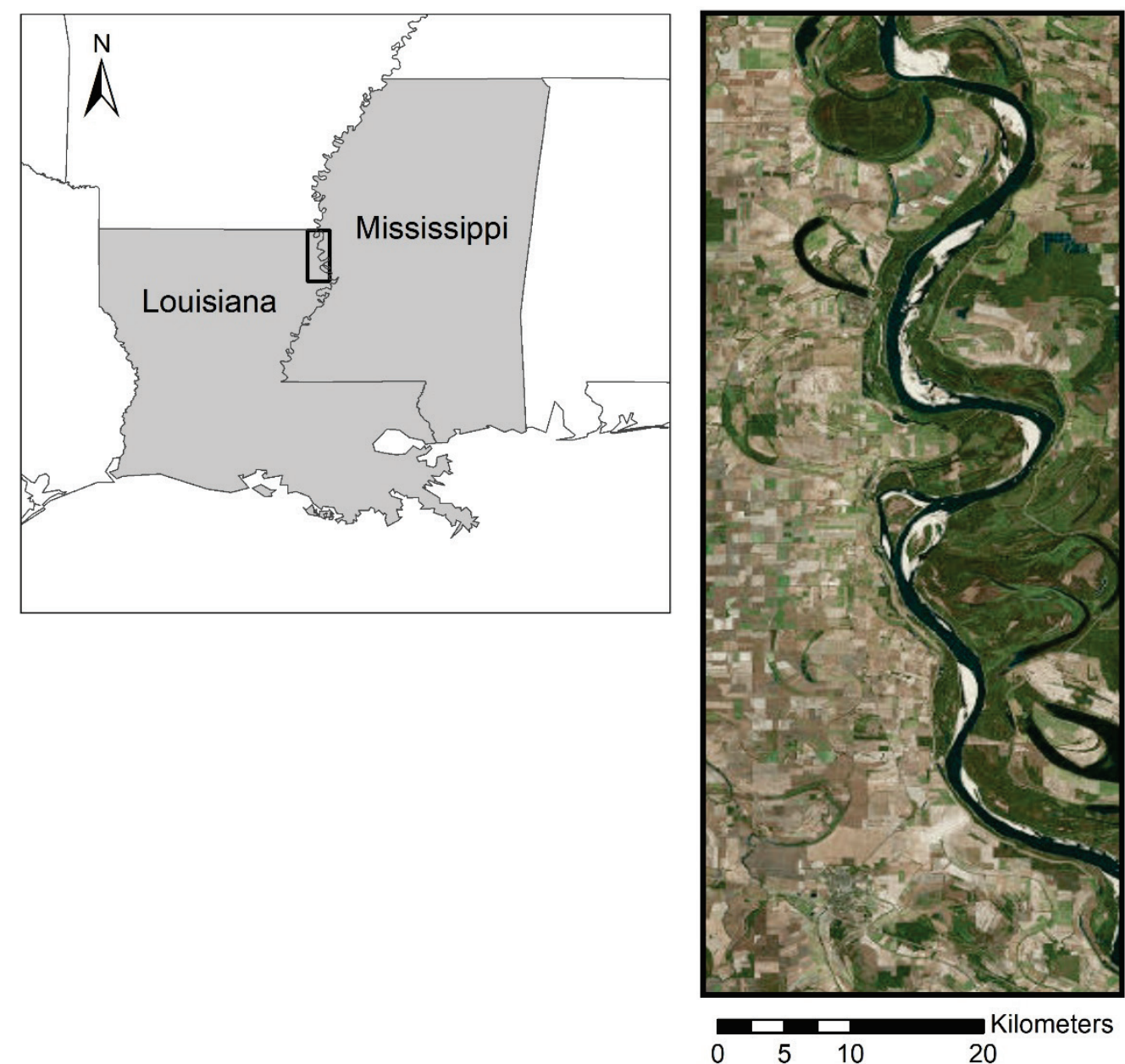

\subsection{Objective}

The objective of this project is to investigate a method for the automatic classification and characterization of ridge-swale patterns along the Mississippi River. The following approach was used to investigate the objective:

1. Create a workflow for classification of ridge-swale patterns using DEMs.

2. Create a workflow for determination of angles of orientation between ridge-swale patterns and the corresponding levee. 
3. Integrate these two workflows to create an automated, comprehensive procedure for the support of subsequent analysis of BEP as part of levee risk analyses.

\subsection{Approach}

The research objectives are addressed through development and application of both machine learning and image-processing techniques. First, the necessary DEM data are acquired. These data are then used to establish the training data set. Next, two machine learning algorithms (i.e., convolutional neural network and U-net) are implemented and assessed. The results are then passed through a series of image-processing steps to identify linear features and trends. Finally, the outputs are organized and assessed to determine the effectiveness of the workflow. 


\section{Procedure}

\subsection{Data acquisition}

DEMs provide a useful data source for identifying ridge-swale systems given their unique pattern of topographic relief. For this study, DEMs are sourced from the USGS National Geospatial Program (USGS 2017).

The spatial resolution of the data set is $1 / 3$ arc-sec (approximately 10 $\mathrm{m}$ ), which is sufficient for the identification of smaller-scale features, such as ridges and swales. The elevation data are provided in meters and, over the conterminous United States, are referenced to the North American Vertical Datum of 1988 (NAVD 88). The vertical resolution of the data is $1 \mathrm{~m}$, which is fine enough to distinguish the elevation differences in ridge-swale patterns.

The information is then trimmed to the study area identified in Figure 2 to remove excess data and better focus on space bounding the Mississippi River and associated artificial levee structures.

\subsection{Classification of ridge-swale patterns}

\subsubsection{Processing}

To implement the classification scheme, a labeled data set is required. In this case, the labeled data set is derived by visual interpretation. The DEM is inspected for the characteristic undulating linear morphology of ridges and swales. Swales are then labeled by digitizing a line down the center of the swale trough. Figure 3 depicts 757 swales interpreted within the study area. Subsequent processing is performed on this data set to create data labels compatible with each of the classification schemes. 
Figure 3. DEM of study area (Figure 2) containing interpreted swales (red lines).

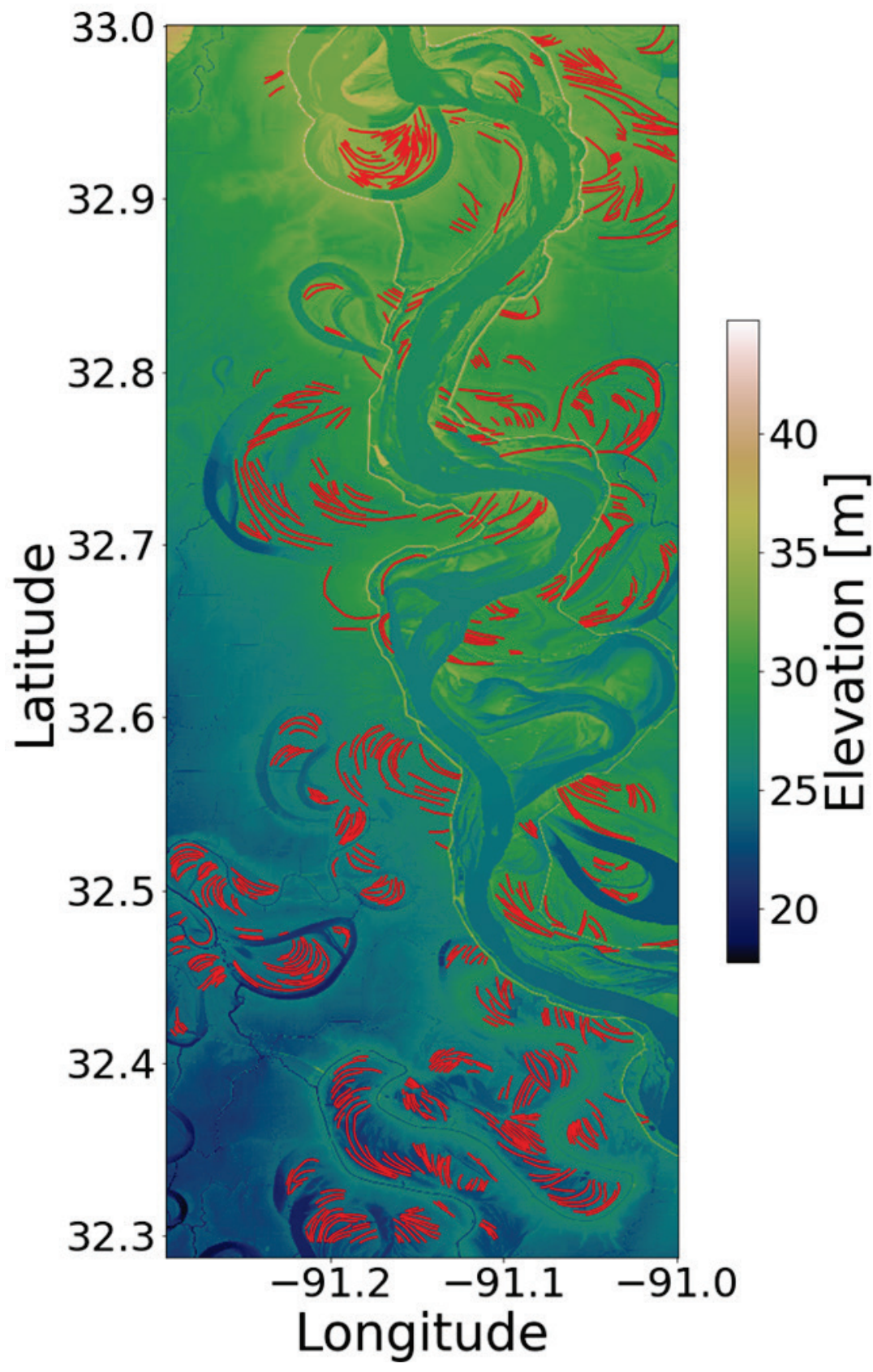

\subsubsection{Classification using a convolutional neural network}

The first machine learning classification algorithm investigated is a convolutional neural network (CNN). This is a type of deep-learning algorithm frequently implemented in image classification tasks because it is able to "successfully capture the spatial and temporal dependencies in an image through the application of relevant filters" (Saha 2018). The algorithm does so by assigning weights and biases to various features in 
the image. It then updates these weights by comparing them to the class label of an image to produce a trained model.

CNNs perform image-wise classification; therefore, they require individual images with labels as their input data. Thus, the labeled DEM containing the 757 swales is made into individual images, each with an associated class label. To accomplish this, the polyline shapefiles are interpolated to map the location of the swale features to the associated grid values. This allows for nearly all the pixels in the DEM grid that contain the swale shapefile to have a corresponding point. Figure 4 shows an example of the interpolation.

Figure 4. A depiction of the interpolation of interpreted swales (left) represented as red lines versus corresponding discretized grid values (right) represented as individual pixels shown in gray.

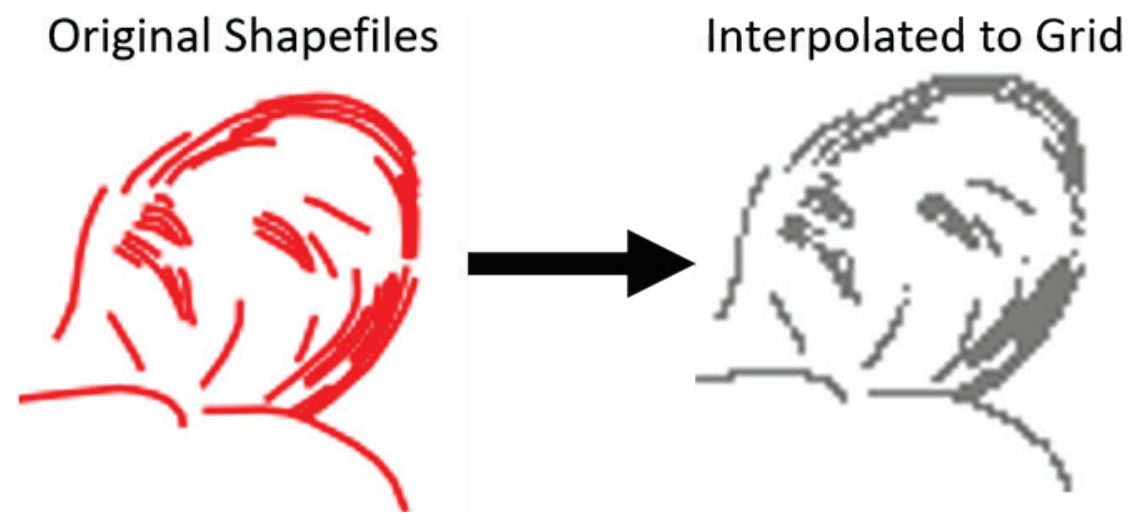

Once the interpolation is achieved, the DEM and the interpolated swale grid are then subdivided into 2,232 sections. Only two class labels are used to automate the labeling of these images and avoid time-consuming manual labelling of every image: 0 for non-swale images, and 1 for swaleimages. If the swale grid corresponding to one of the sub-divided DEM images contains at least one swale pixel, it is assigned a class label of 1 . If the swale grid corresponding to the sub-divided DEM images contains no swale pixels, it is assigned a class label of $o$. Various thresholds are also used to try and distinguish images with a greater number of swale pixels (e.g., setting the class label to 1 only if the image contained greater than 10 swale pixels) to see if this has an impact on prediction results.

Once the DEM images are given class labels, a convolutional neural network is used to train a model. Results produced by this model are presented in Section 3.1.1. 


\subsubsection{Classification using a U-net}

The second machine learning classification algorithm investigated is a Unet. The U-net architecture takes advantage of convolutional neural network model but implements it in a slightly different structure (Lamba 2019). In the case of the U-net, the result is pixel-wise classification, as opposed to image-wise classification of the traditional CNN architecture.

Because the U-net is designed to perform pixel-wise classification, each pixel of the DEM is assigned a class label. The resulting classes are contained in an image format, commonly referred to as a "mask." In this implementation, a swale mask is constructed in two steps. First, each of the interpolated grid values from the shapefile is assigned a class label of 1 . Second, a Gaussian smoothing filter with thresholding is applied to correct for potential gaps in the mask or misalignments with the swale feature. The result is more continuous, fuller-coverage mask labels. An example of this process is shown in Figure 5 .

Figure 5. DEM (left) and associated masks after swale interpretation (middle) and Gaussian filter with thresholding (right).
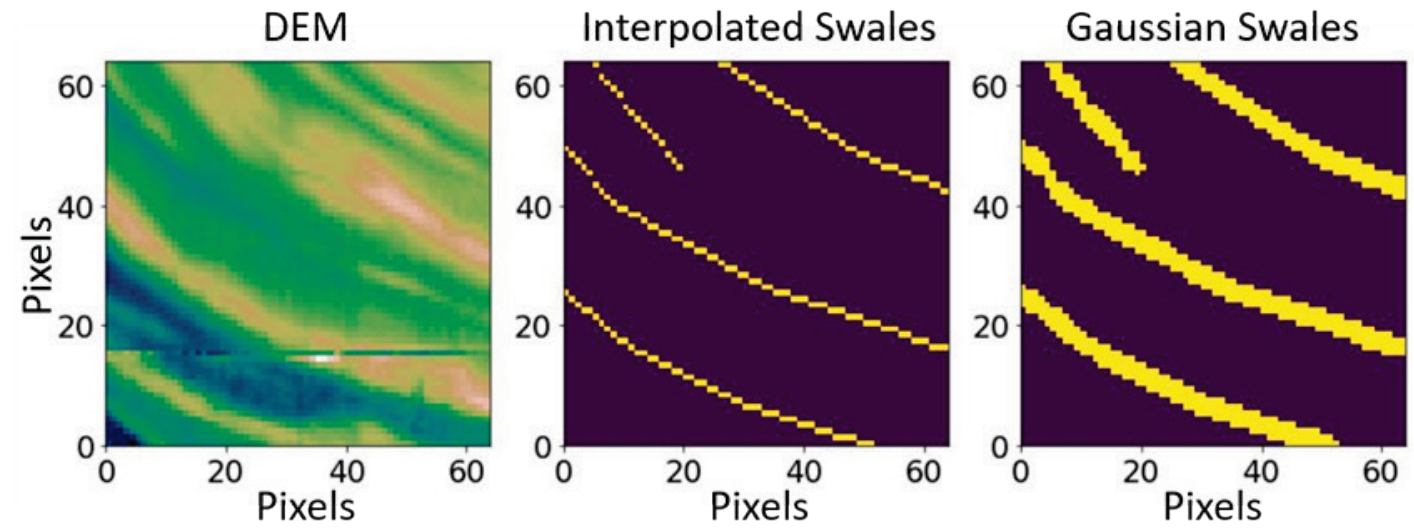

Once these masks are created, the U-net algorithm is applied to train a model. The results are discussed in Section 3.1.2.

\subsection{Characterization of ridge-swale patterns}

While the previous sections outline approaches for automated classification of swales, it is also of interest to automatically detect the swale pattern orientation in relation to corresponding levees. This section describes a proposed procedure for such an analysis. 


\subsubsection{Highlighting linear features}

As ridge-swale patterns can appear as linear features in DEMs, the DEM is first divided into smaller sub-images. Once the larger region is subdivided, a series of three processing steps is applied to the data. Because ridges are relatively higher elevations than swales, taking the gradient of an image highlights the boundary between adjacent ridges and swales, as this constitutes a relatively rapid change in elevation. Thus, the first step is to take both horizontal and vertical derivatives of each image. From these values, the magnitude of the gradient is computed using the following relationship:

$$
\|\nabla G\|=\sqrt{G_{x}^{2}+G_{y}^{2}}
$$

where:

$$
\begin{aligned}
\|\boldsymbol{\nabla} \boldsymbol{G}\| & =\text { magnitude of the gradient } \\
\boldsymbol{G}_{\boldsymbol{x}} & =\text { horizontal derivative of image } \\
\boldsymbol{G}_{\boldsymbol{y}} & =\text { vertical derivative of image }
\end{aligned}
$$

An example of a gradient calculation performed on a ridge-swale pattern is shown in Figure 6. Figure 6(a) shows the original section of elevation data containing a ridge-swale pattern. Figure 6(b) shows the magnitude of the gradient of the original data. 
Figure 6. A depiction of the spatial domain processing steps implemented on the DEM data set.
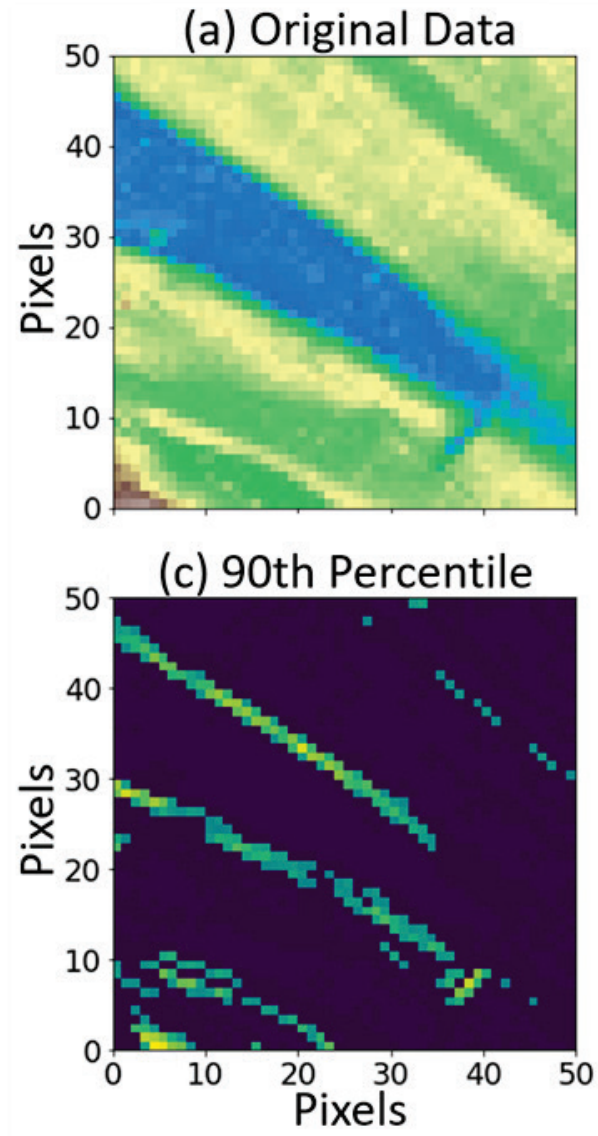

(b) Gradient

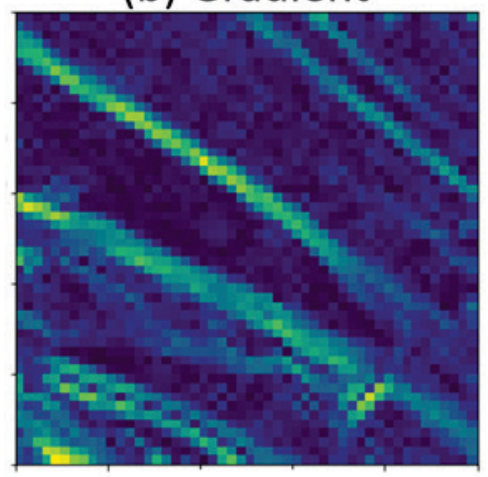

(d) Median Filter

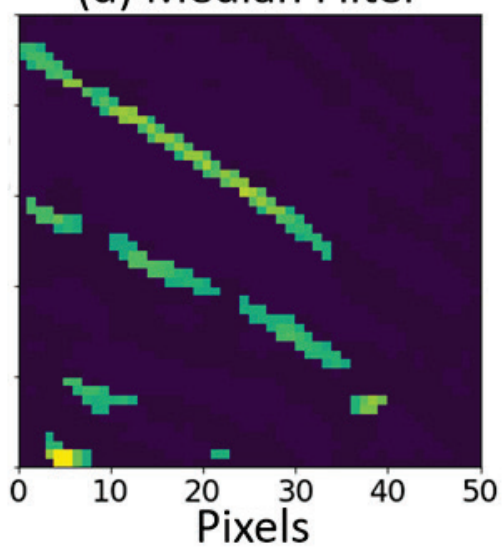

The second processing step involves removing low magnitude values of the gradient. This eliminates data points representing areas of relatively small changes in elevation to highlight the undulating ridge-swale topography. This is accomplished by calculating the $90^{\text {th }}$ percentile value of the magnitude of the gradient and assigning all data points that fall below this threshold a value of zero. An example of this can be seen in Figure 6(c). This results in areas of large gradients being preserved.

Though the linear features are significantly more pronounced after removing the bottom 9oth percentile of gradient values, there are still areas of discontinuity between neighboring data points. Ridge-swale patterns are typically characterized by distinct, continuous boundaries between areas of high and low elevation. Thus, in the third processing step, a median filter is applied to better distinguish the continuous gradient values in the data and further remove areas of discontinuous gradient values. An example of these filtering results is shown in Figure 6(d). 


\subsubsection{Application of the Hough transform}

Once the linear features are adequately distinguished, the Hough transform is applied. This transform is commonly used for feature detection in image-processing applications and is applied in this workflow as a method of identifying linear features in images.

The Hough transform makes use of the normal parameterization of a line, which makes it particularly useful for this application. In this parameterization, a line can be described in the $x y$-plane by its distance $(\rho)$ and angle $(\theta)$ parameters through the following relationship:

$$
\rho=x \cos \theta+y \sin \theta
$$

where:

$$
\begin{aligned}
\boldsymbol{\rho}= & \text { distance from origin to nearest point on the line } \\
\boldsymbol{x}= & \text { horizontal position } \\
\boldsymbol{y}= & \text { vertical position } \\
\boldsymbol{\theta}= & \text { angle between the horizontal } \mathrm{x} \text {-axis and the line connecting } \\
& \text { the origin and the nearest point }
\end{aligned}
$$

In practice, the transform essentially loops through various angles in an image and returns the $(\rho, \theta)$ parameters of the line that yield the dominant linear trend. This allows for the determination of angles of linear features in the processed DEM sub-images.

The implementation of the transform is straightforward in Python. For this implementation, two functions from the skimage processing library are used: hough_line and hough_line_peaks. With these functions, the Hough transform is applied and the maximum intensity trend line determined. The trend line is then plotted on the original data.

The Hough transform provides two key benefits towards the project goals. First, plotting trend lines provides a visual output that may be compared against the original swale interpretation and DEM. Second, the transform returns an angle for use in subsequent spatial and orientation analyses between swales and other elements, such as a levee structure. An example of the application of the Hough transform is shown in Figure 7. Figure 7(a) shows data filtered using the processing steps described in Figure 6. Figure 7 (c) depicts the filtered data after applying the Hough transform. 
The data are plotted as angle versus distance. Note that areas of intersection indicate the most prevalent orientation of linear features observed in the data. Figure 7 (b) shows the maximum trend line determined by the highest intensity angle in Figure $7(\mathrm{c})$. The trend line follows the ridge-swale boundary almost exactly.

Figure 7. An example of the Hough transform applied to a filtered section of data containing a ridge-swale pattern.
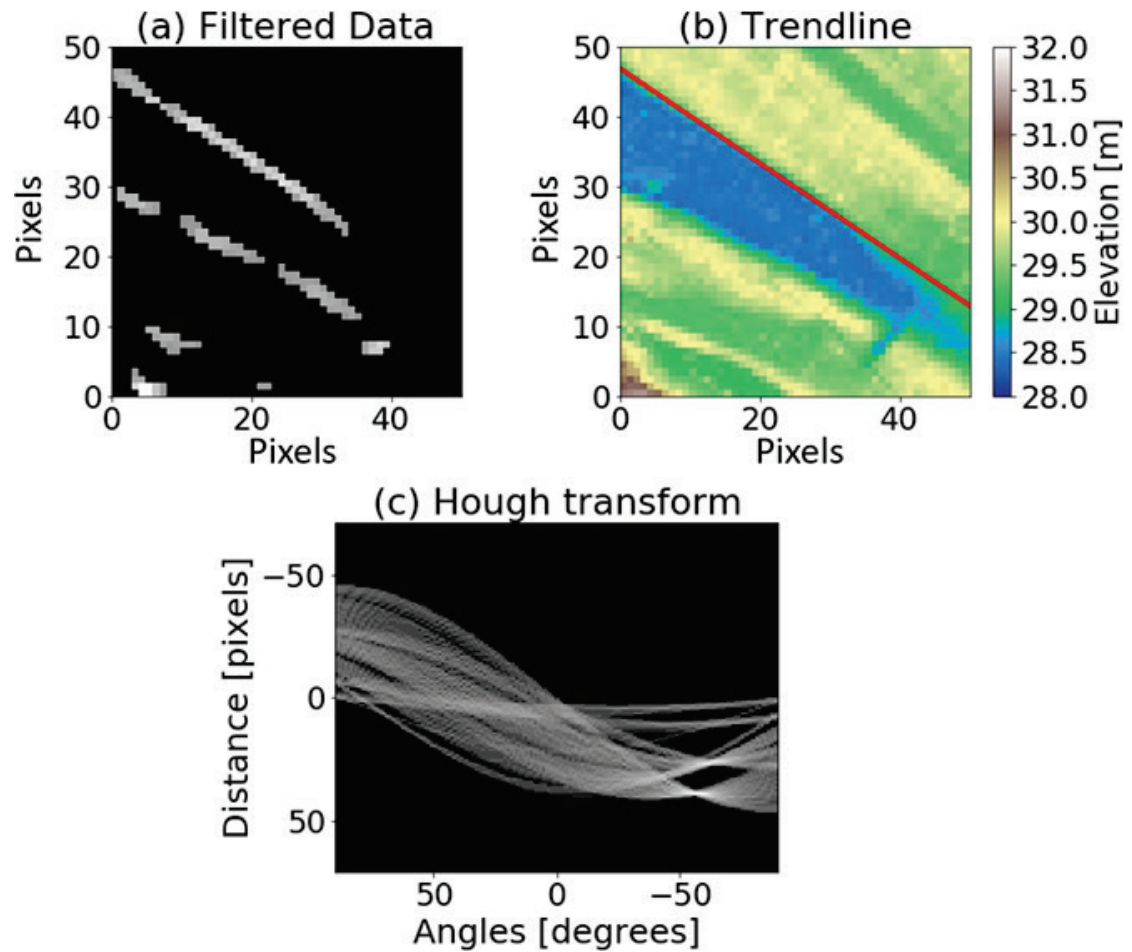


\section{Results and Discussion}

\subsection{Results}

\subsubsection{CNN classification}

Classification using a CNN did not yield meaningful results. The algorithm delivered appreciable accuracy above 70 percent but on further investigation, the model was predicting only a single class and was incapable of distinguishing images that contained swales. One explanation is that the number of images with a o class label (containing no swales) was significantly larger than the number of images with a 1 class label (containing swales). Thus, the highest accuracy the model could produce was achieved with predicting all images as class 0 .

This issue could be investigated and addressed through a reduction in the number of class 0 , non-swale images used to train the model. For example, the study area could be further clipped to remove excessive non-swale regions farther from the banks of the Mississippi River. This may produce more meaningful results. However, this was not tested because the U-net approach proved a more favorable alternative to the $\mathrm{CNN}$ method.

\subsubsection{U-net classification}

Using a U-net approach was determined to be more favorable than a CNN approach for swale classification. One reason for this was the aspect of pixelwise classification because it provides the ability to not only identify an area in which a ridge-swale pattern exists, but to also identify where exactly that pattern is located within an image. Additionally, the model trained using a Unet approach yielded results that indicated an ability to distinguish swale features from non-swale features, whereas the model trained using a CNN did not distinguish features. However, despite improved results over the CNN model, the U-net model results were still not determined to be sufficient for operational use for reasons discussed in the paragraphs that follow.

The final U-net model prediction results were varied. Three general cases were observed. In the first case, it appeared that the model was able to accurately determine where swale features were in an image. Masks for these images were reasonably well-aligned with the corresponding swale features. In the second case, the model was unable to identify regions corresponding to swale masks, instead identifying 
non-swale features that exhibited elements of morphology similar to the ridge-swale pattern but were not interpreted as such. In the third case, swale and non-swale features were not identified at all, and no distinguishable prediction was made by the model.

An example of each case is shown in Figure 8. The panels in the left column represent DEM images. The panels in the middle column contain swale masks with yellow pixels representing a class label of 1 (swale pixel) and purple pixels representing a class label of o (non-swale pixel). The panels in the right column show the prediction results from the trained model with yellow pixels representing relatively higher probabilities of being swales and blue and purple pixels representing lower probabilities. Row (a) depicts promising results, where the yellow regions of higher intensity correspond well with the swale masks and areas of low elevation in the DEM. Row (b) shows slightly less favorable results. The horizontal feature in the image appears to have been recognized by the model, but it failed to predict the presence of the interpreted swale in the bottom right corner of the image. Row (c) shows no distinguishable results. Note that the masks created do not exactly align with distinguishable swale features in the actual DEM image. This could potentially contribute to the lower prediction accuracy observed. It is possible that the DEM accuracy was also limited by the elevation contrast. Convolution operations would further blur these features, resulting in limited contrast for detection neurons in the algorithm. With higher resolution DEM data, it is possible that this approach would work quite well. 
Figure 8. Three different prediction results from the model trained with a U-net with the original DEM (left column), swale masks (middle column), and prediction results from the trained model (right column).

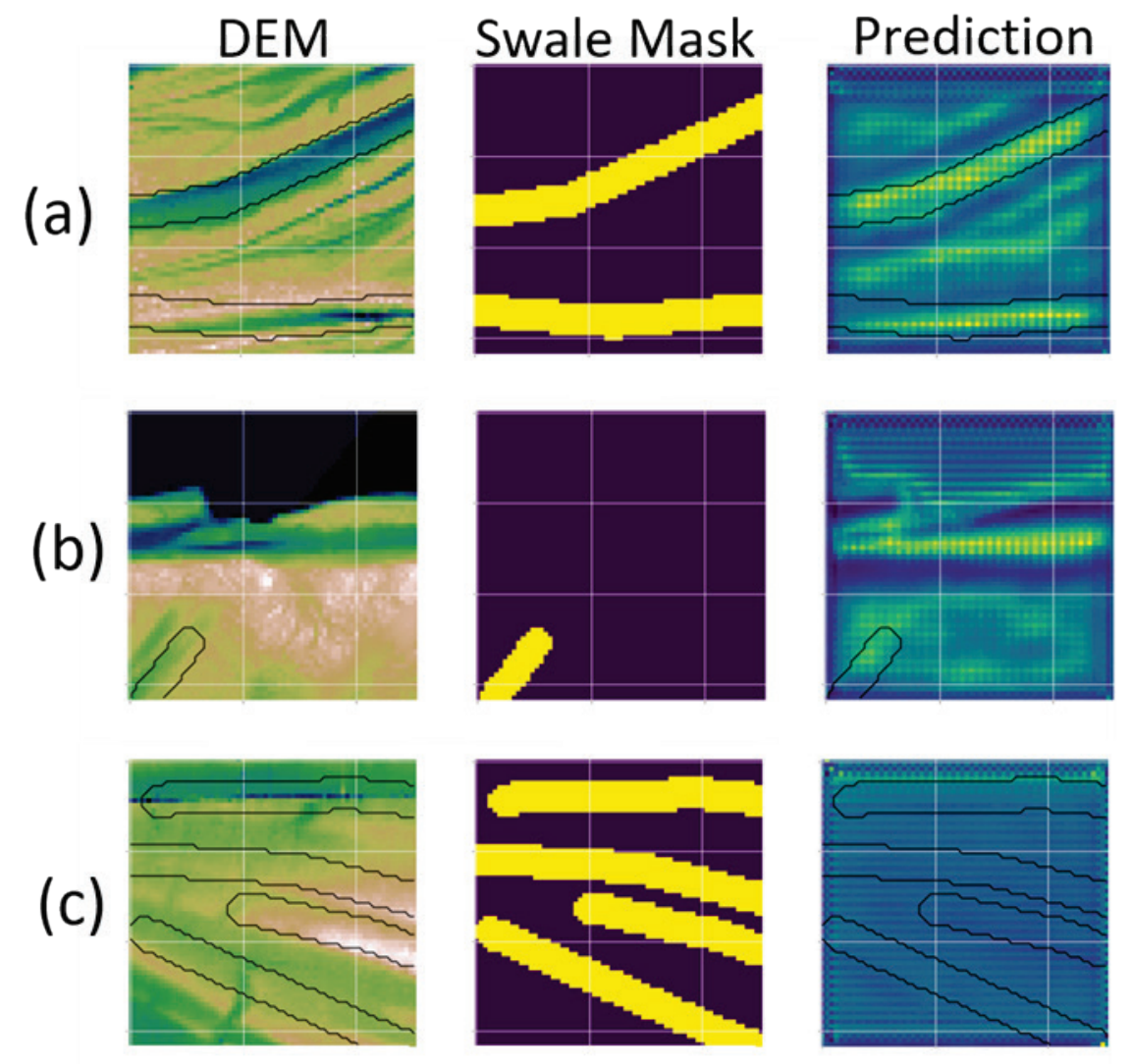

The procedure used to create the image masks likely had a negative effect on result accuracy. A clear instance of this can be observed in Figure 8(c). In the DEM, it is unclear whether a swale feature exists (compare to the masked features in Figure 8[a]) despite four interpreted swale features indicated in the image mask. It may also cause some features that are swales to be misidentified as non-swales. This unfavorable effect is a result of the interpolation and filtering used to create the image masks. In the original labeling of the DEM, swales were identified using shapefiles that are not directly transferable to image masks. Thus, the "rough-but-fast" method used to create masks from the shapefiles themselves likely limited the accuracy of the results.

\subsubsection{Ridge-swale orientations}

The final step of the characterization workflow involved assigning a measure of orientation to identified swale features. Figure 9 depicts a region that was divided into 36 sub-images to complete the full characterization 
workflow. The results shown in Figures 9 and 10 are promising. First, the image displays that many of the ridge-swale boundaries are identified, along with the associated angles. The levee was also distinguished exceptionally well. This makes sense as the algorithm is designed to identify dominant linear trends. As a result, if multiple linear features, such as a swale and levee structure, occur within a sub-image, only the dominant linear trend will be identified. Minimizing sub-image size or masking unwanted features exhibiting strong linear trends can reduce suppression of swale responses.

Figure 9. DEM where the characterization workflow has been applied to subsections to plot the dominant trend lines (red).

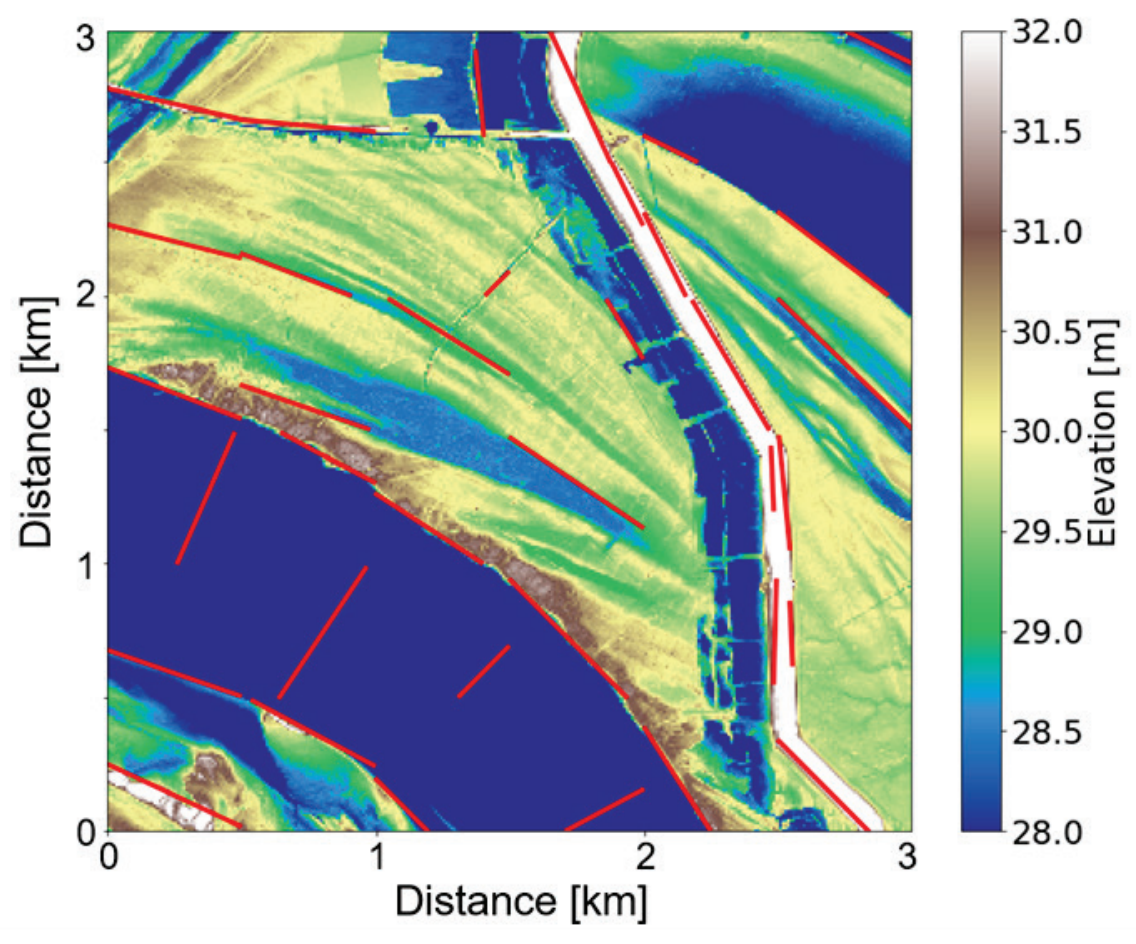


Figure 10. DEM and cross section with intersecting dominant linear trends.

Ridge-Swale Region along the Mississippi River
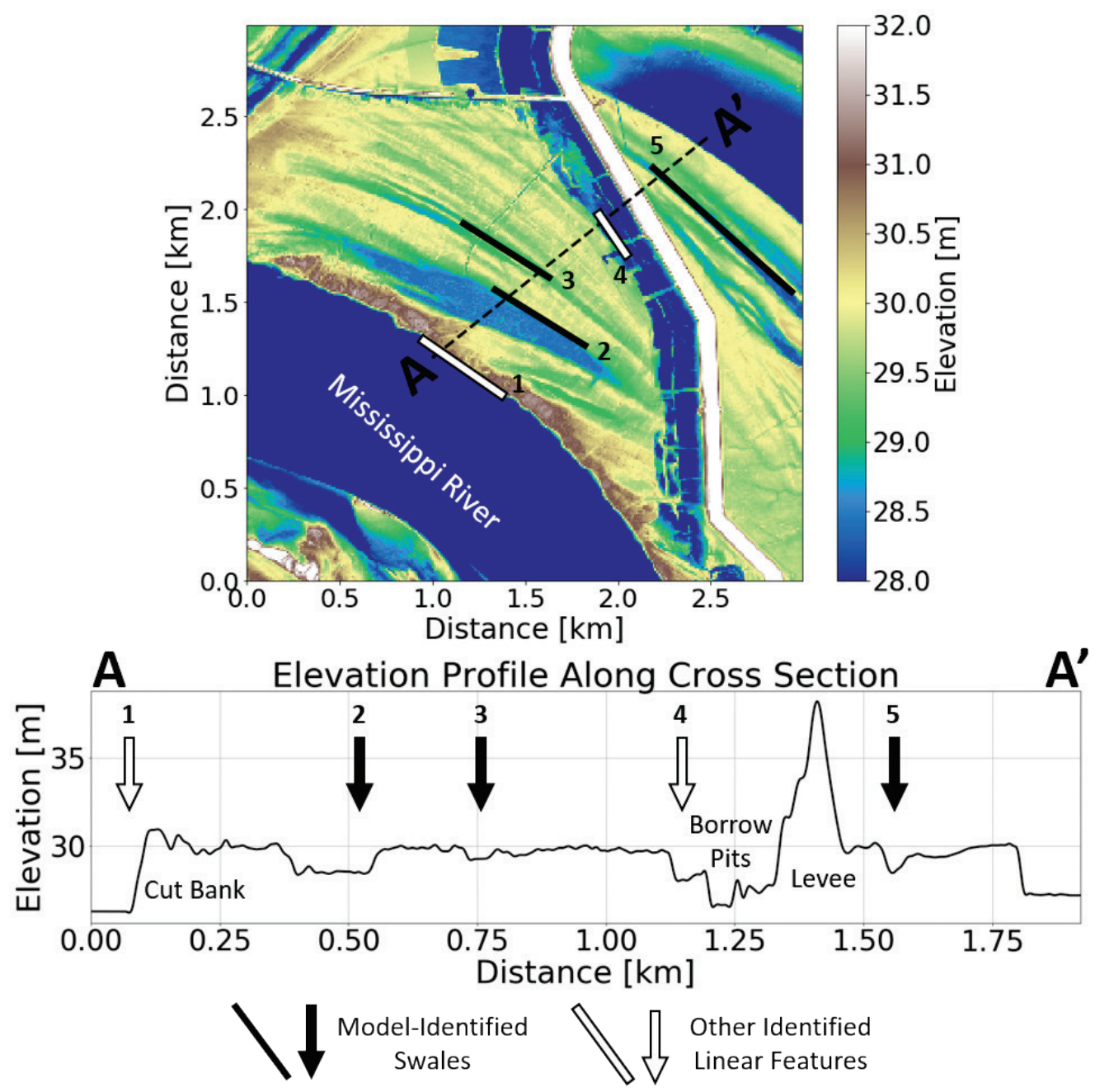

This result has a practical application in helping determine the angles of orientation between swales and adjoining levees. Because this workflow was able to plot the trend lines and return angles of swales and levees, determining the orientations between these features should be straightforward.

Another interesting response from Figure 9 is the trend lines plotted within the river and along the riverbanks. These trend lines do not provide any meaningful information but do help in highlighting a key characteristic of this workflow. The methodology does not have the ability to interpret features; it merely plots the dominant linear trend observed in the data. Though it can "find" the boundaries between ridges and swales, this is a result of the processing steps integrated into the workflow. To 
become more useful in determining orientations of ridge-swale patterns specifically, the classification scheme must be integrated to initially identify where these patterns exist in a DEM. 


\section{Conclusions and Recommendations}

\subsection{Conclusions}

The Mississippi River is the main channel in part of a large, dynamic drainage basin in the center of the continental United States. The dynamic nature of this system, along with its many tributaries, lends itself to frequent flooding events. Without the current man-made structures in place, one of which is a levee, these flooding events would devastate local infrastructure along the river. Thus, it is essential that the integrity of these structures be maintained. Current methods in place to evaluate maintenance primarily include manual inspections, which are timeconsuming and inefficient. Though these manual inspections provide valuable, in-depth information, they generally assess only where maintenance is currently required and do not anticipate where maintenance may be needed in the future. Thus, there is a need for a more automated, predictive method to determine possible areas of failure in these structures before a failure occurs.

It has been shown that the orientation of ridge-swale patterns with levees can lead to sand boils and possibly BEP, which can compromise the integrity of levees and possibly lead to failure. Thus, the successful implementation of the outlined approach to automatically classify and characterize ridge-swale patterns in DEMs would provide an efficient method for determining a key predictor of levee failure, helping to improve the current measures already in place and to reduce the harmful effects of flooding events along the Mississippi River.

While the results of this project are promising, in their current state the workflows are not yet operational. The first step in the approach of the project was to create a workflow for the classification of ridge-swale patterns using DEMs. A U-net pixel-wise classification scheme was used to train a model, the accuracy of which would need to be improved before it can be implemented for practical use. However, some of the prediction results showed the ability of the model to distinguish between swale and non-swale features. Further tuning of the model and input data would likely increase its prediction accuracy, possibly to an appropriate level for operational implementation. The second step of the project was to create a workflow for determination of angles of orientation between ridgeswale patterns and the corresponding levee. The workflow created to 
accomplish this proved effective in identifying linear trends in DEMs and can return angles of the associated trend lines. In particular, the workflow was able to identify linear trends of both levees and ridge-swale features in DEMs, a useful step in fulfilling this objective; in its current form, the workflow does not return angles of orientation between features. The third step was to integrate the two workflows to create an automated, comprehensive procedure for the support of subsequent BEP hazard analysis. With the successful implementation of the classification and characterization workflows along with the proper integration with engineering applications, this objective is attainable. Recommendations for how to accomplish this are discussed in the following section.

\subsection{Recommendations}

One of the key faults in the implementation of the U-net was the process of creating image masks. Certain features were masked that did not represent swales, while other swale features were not masked. Implementing a more thorough labeling of swale features would aid in the improvement of this process. This could be achieved using polygon instead of polyline shapefiles in ArcGIS to more accurately and completely mask swale features. Another area of improvement could come from the integration of additional data types with the DEM data. Using normalized difference vegetation index (NDVI) or other supplemental data to further assist in either identifying swale or nonswale features may be another avenue worth pursuing.

The angle characterization workflow needs development. While the workflow can determine feature orientations, the outputs do not yet include angles of orientations between swale and levee features and are not optimized for use by a specific end-user. Implementing the workflow as an ArcGIS tool with associated output objects would be a step toward increasing the ease of use and accessibility to geologists and engineers who are likely to be the ones performing posterior analysis.

Once the classification and characterization are working as intended, the final step would be integrating them. The machine-learning classifier would identify areas in a DEM that resemble ridge-swale features, then these areas would be directly fed into the angle characterization workflow. Finally, the outputs could be passed on in an appropriate format for further spatial assessment. 


\section{References}

Bonelli, S (ed). 2013. Erosion in geomechanics applied to dams and levees. New York: Wiley.

Glynn, E., M. Quinn, and J. Kuszmaul. 2012. Predicting piping potential along middle Mississippi River levees. $6^{\text {th }}$ International Conference on Scour and Erosion. Paris: Société Hydrotechnique de France.

Kolb, C. R. 1975. Geologic control of sand boils along Mississippi River levees. AEWESMISC-PAPER-S-75-22. Vicksburg, MS: U.S. Army Engineer Waterways Experiment Station.

Lamba, H. 2019. Understanding semantic segmentation with UNET: A salt identification case study. https://towardsdatascience.com/understanding-semanticsegmentation-with-unet-6be4f42d4b47.

Lobeck, A. K. 1939. Geomorphology, an introduction ot the study of landscapes. New York: McGraw-Hill Book Company.

Mansur, C. I., and R. I. Kaufman. 1957. Underseepage, Mississippi River levees, St. Louis District. Transactions of the American Society of Civil Engineers 985-1008.

Saha, S. 2018. A comprehensive guide to convolutional neural networks - the ELI5 way. https://towardsdatascience.com/a-comprehensive-guide-to-convolutionalneural-networks-the-eli5-way-3bd2b1164a53.

Semmens, S. N., W. Zhou, B. K. Wesenbeeck, and P. M. Santi. 2017. Application of multiple criteria decision making model for evaluation of levee sustainability. Environmental and Engineering Geoscience 65-78.U.S. Army Corps of Engineers (USACE). 2004. The Mississippi River and tributaries project. https://biotech.law.lsu.edu/maps/mrtp/mrtp.htm.

U.S. Environmental Protection Agency (EPA). 2016. The Mississippi/Atchafalaya River Basin (MARB). https://www. epa.gov/ms-htf/mississippiatchafalaya-river-basinmarb.

U.S. Geological Survey (USGS). 2017. National Geospatial Program. https://www.usgs.gov/core-science-systems/national-geospatial-program. 


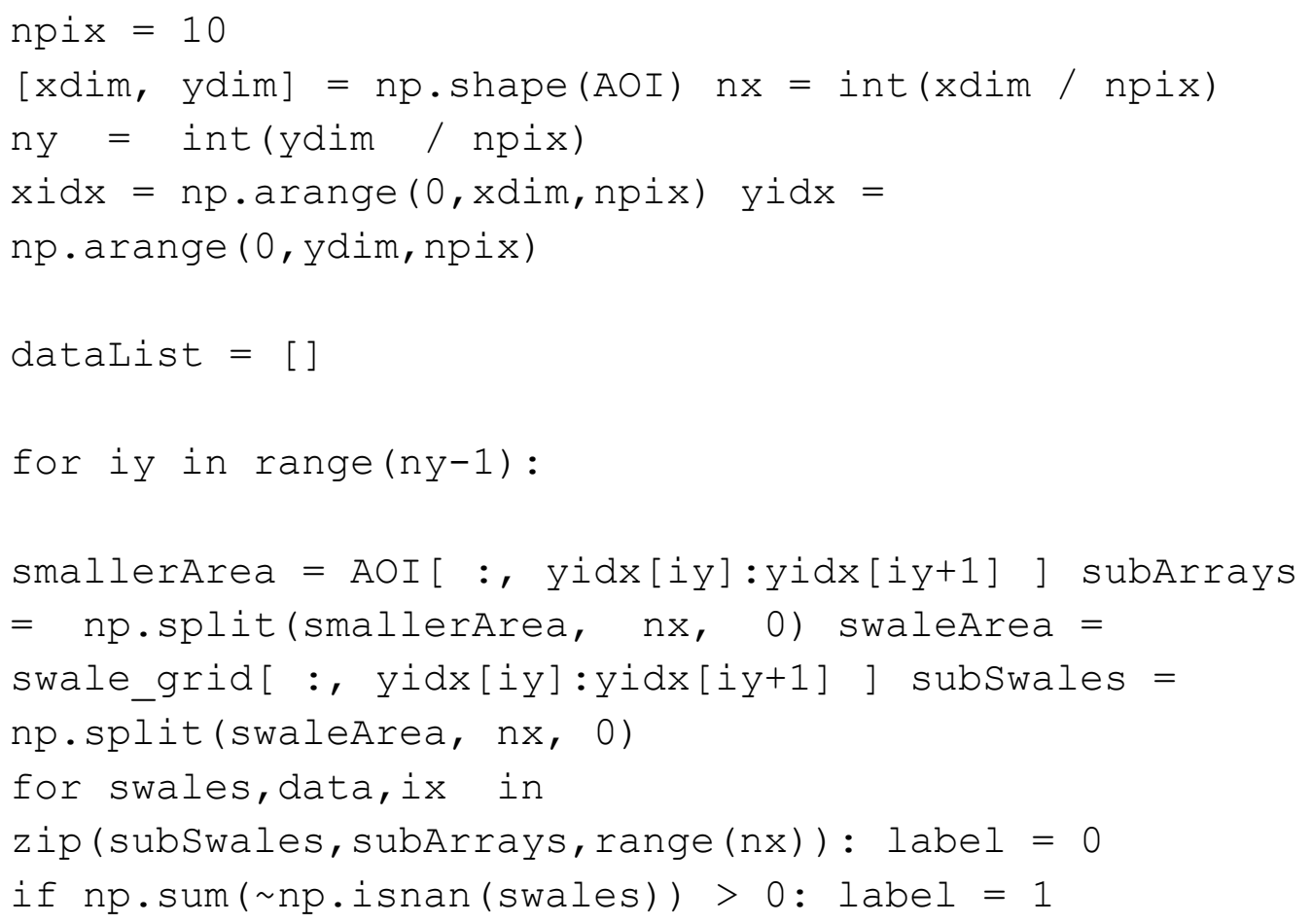




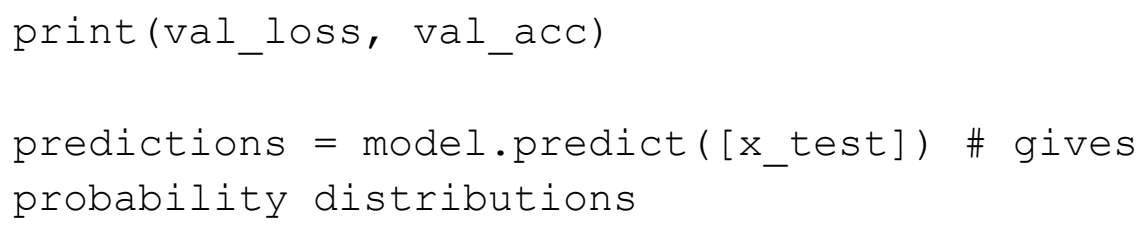

\section{A.2 U-net classification}

import numpy as np import pandas as pd import matplotlib.pyplot as plt from osgeo import gdal from matplotlib import $\mathrm{cm}$ import geopandas as gpd import shapefile as shp \# Requires the pyshp package from scipy.interpolate import interpld from scipy.ndimage import gaussian_filter

\# machine learning libraries import os import random

from tqdm import tqdm_notebook, tnrange from itertools import chain

from skimage.io import imread, imshow, concatenate_images from skimage.transform import resize from skimage.morphology import label from sklearn.model_selection import train_test_split import tensorflow as tf

from keras.models import Model, load_model

from keras.layers import Input, BatchNormalization, Activation, Dense, Dropout from keras.layers.core import Lambda, RepeatVector, Reshape from keras.layers.convolutional import Conv2D, Conv2DTranspose from keras.layers.pooling import MaxPooling2D, GlobalMaxPool2D from keras.layers.merge import concatenate, add from keras.callbacks import Earlystopping, Modelcheckpoint, ReduceLROnPlateau from keras.optimizers import Adam 


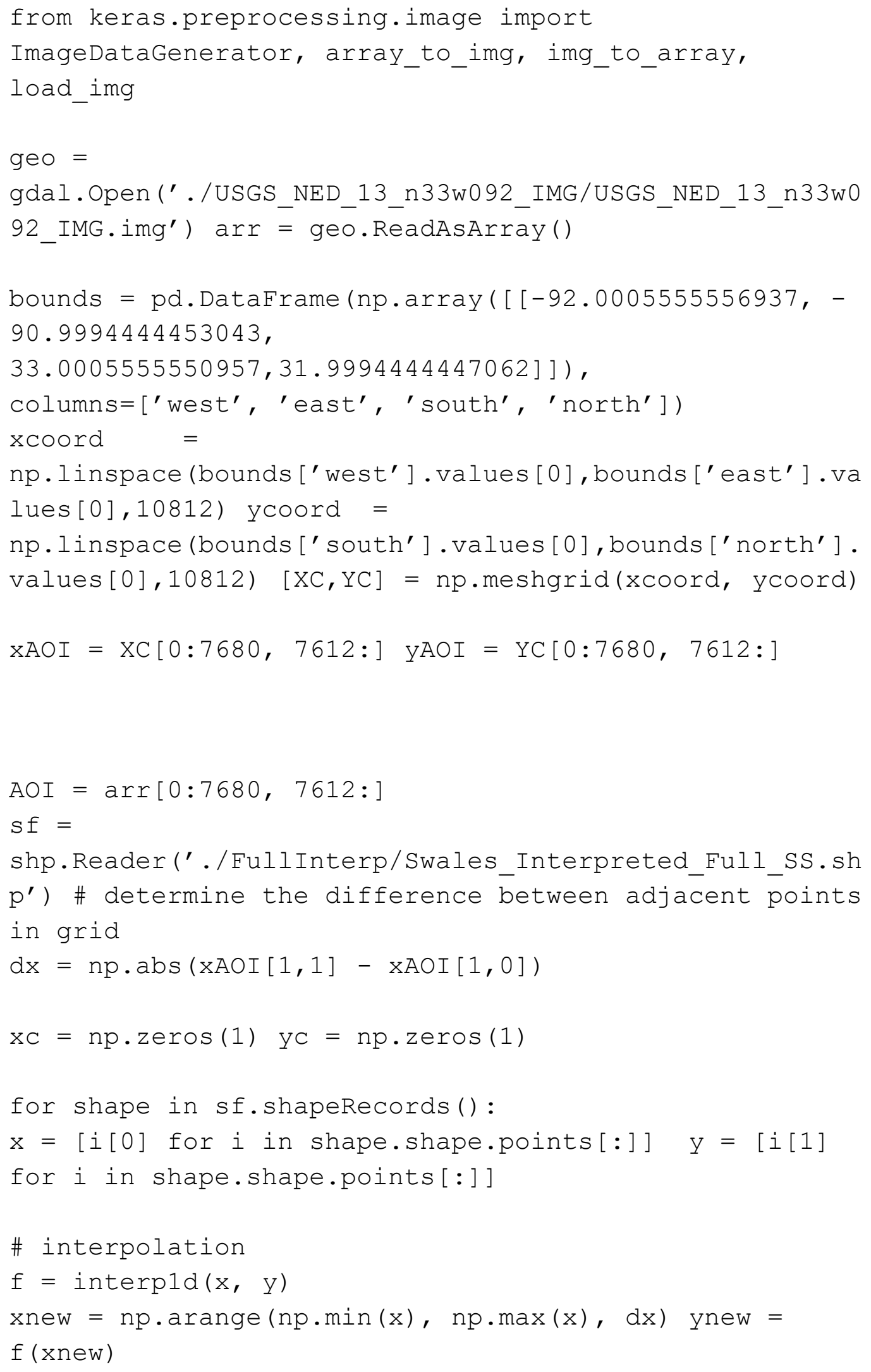




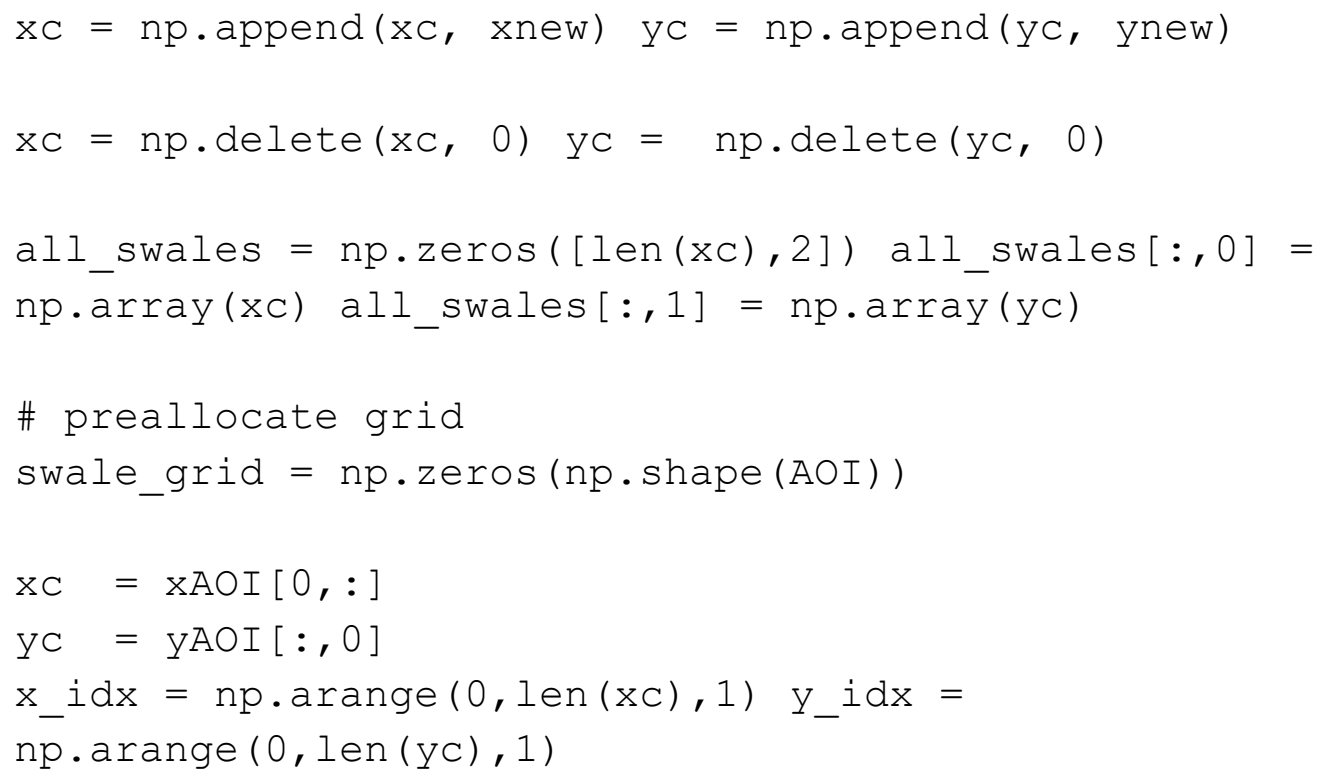




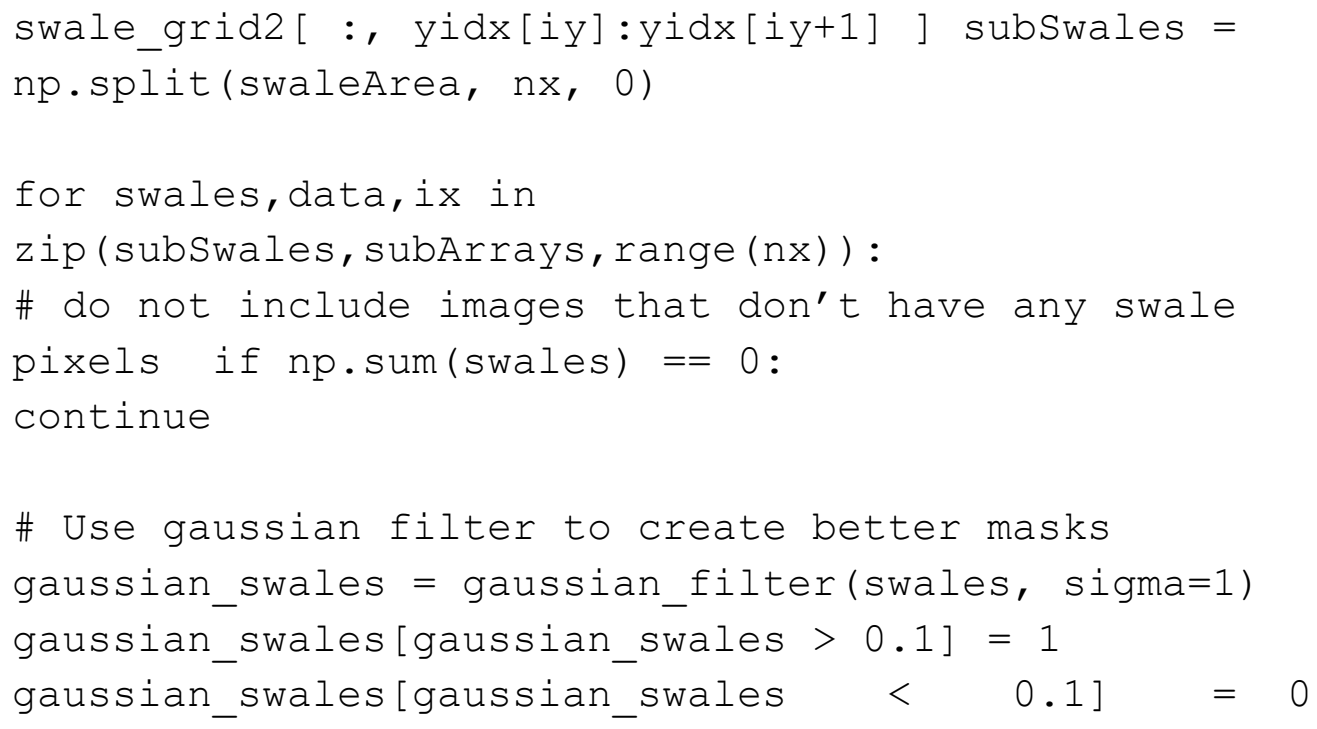




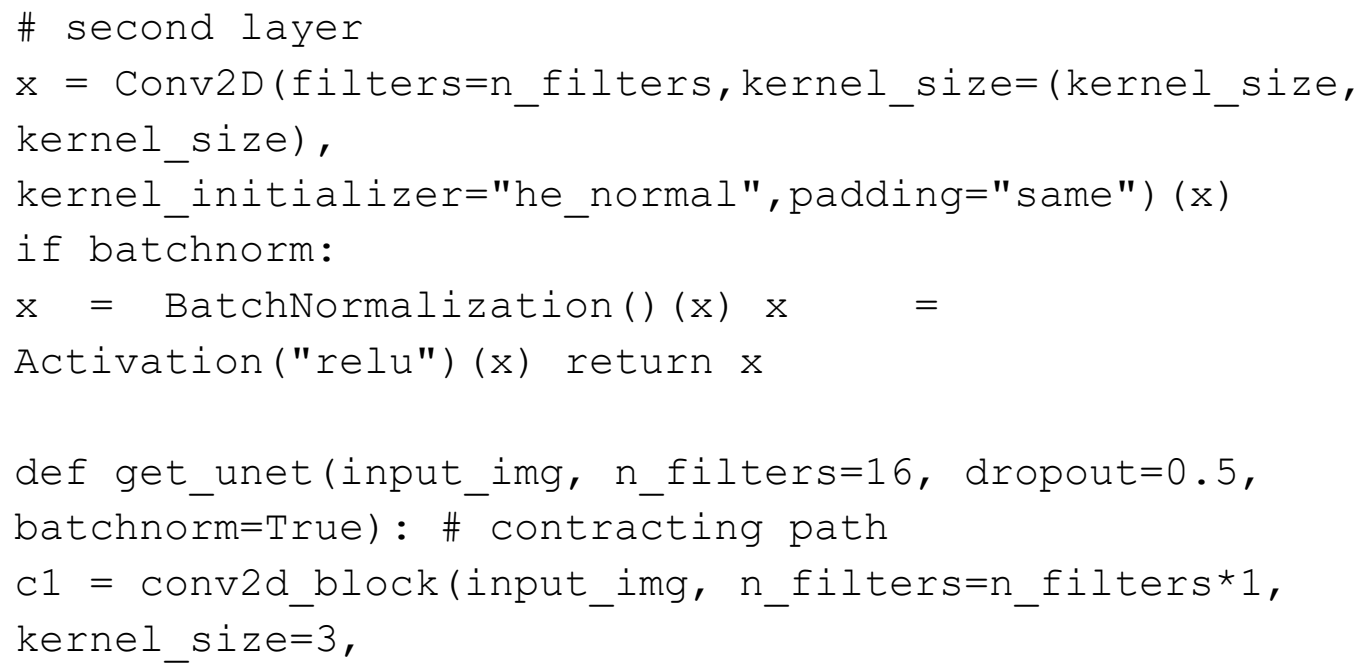




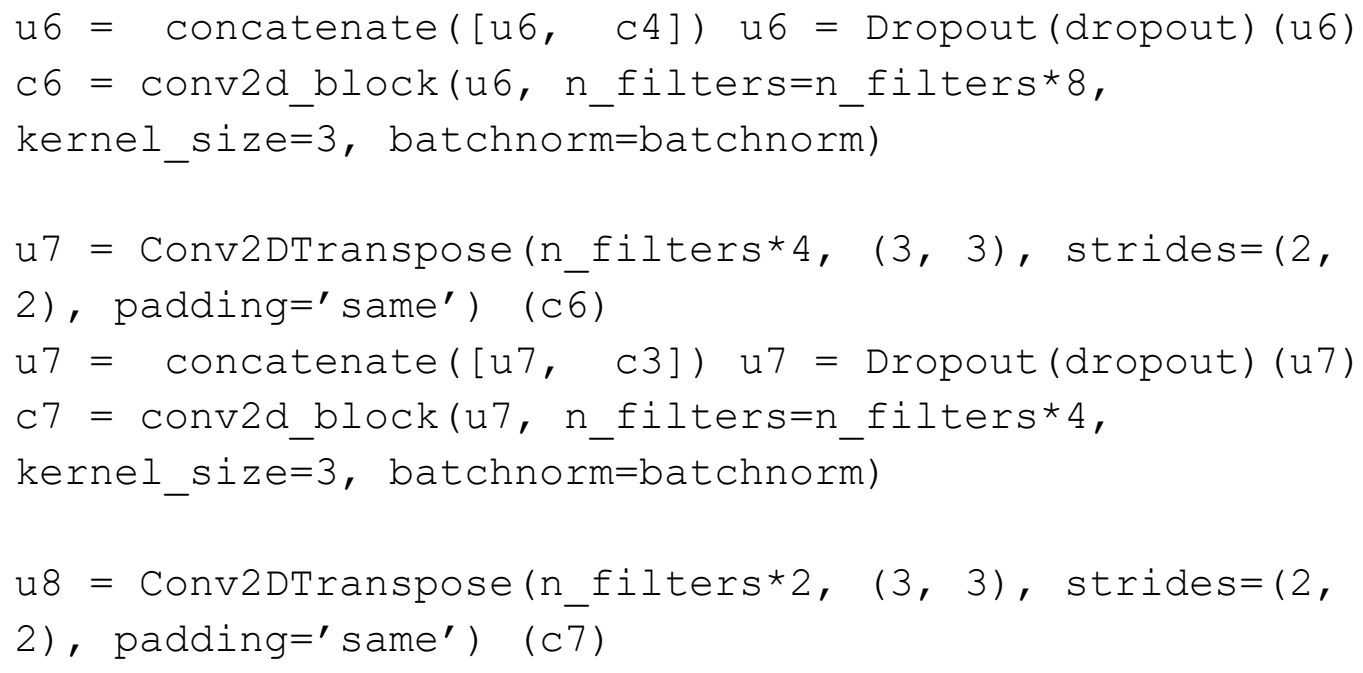


Earlystopping (patience=10, verbose=1),

ReducelRonPlateau (factor=0.1, patience=3, min_lr=0.00001, verbose=1), ModelCheckpoint ('modeltgs-salt.h5', verbose=1, save_best_only=True, save_weights_only=True)]

results = model.fit(x_train, y_train, batch_size=32, epochs=5, callbacks=callbacks, validation_data $=\left(x_{\text {_test }}, y_{\text {_test }}\right)$ )

\# Load best model model.load_weights('model-tgssalt.h5')

\# Evaluate on validation set (this must be equals to the best log_loss) model.evaluate (X_test, y_test, verbose=1)

\# Predict on train, val and test preds_train = model.predict (X_train, verbose $=1$ ) preds_test $=$ model.predict (X_test, verbose $=1$ )

\# Threshold predictions

preds_train_t $=$ (preds_train >

$0.5)$.astype (np.uint8) preds_test_t $=$ (preds_test >

$0.5)$. astype (np.uint8)

\section{A.3 Angle characterization}

import numpy as np import pandas as pd import matplotlib.pyplot as plt from matplotlib import $\mathrm{cm}$ import gdal from scipy import signal from skimage.transform import (hough_line, hough_line_peaks, probabilistic_hough_line)

\# read img file into an array geo $=$ gdal.Open('./USGS_NED_13_n33w092_IMG/USGS_NED_13_n33w0 92_IMG.img') arr = geo.ReadAsArray () 


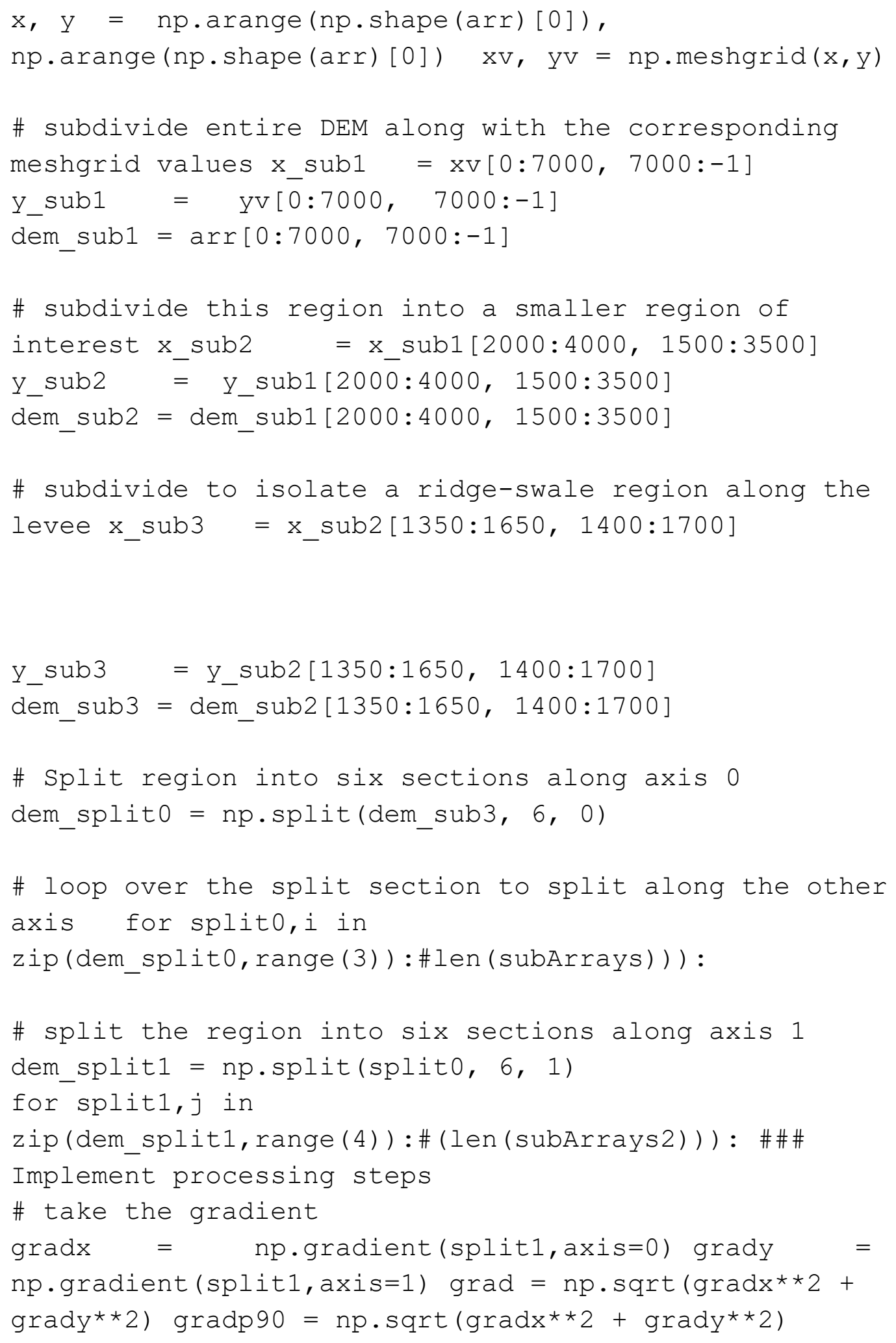


\# set gradient values below the 90th percentile to

0 p90 = np.percentile (np.abs (grad), 90)

gradp90[np.abs (grad) < p90] = 0

\# apply a median filter to the 90th percentile of

gradients medp90 =

signal.medfilt2d(gradp90,kernel_size=3)

\#\#\# Apply the hough filter \# example can be found at $\mathrm{h}$, theta, $\mathrm{d}=$ hough line (medp90) rows, cols $=$ medp90. shape

_, angle, dist $=$ hough_line_peaks (h, theta, d, num_peaks=1) yo $=($ dist $-0 \star n p \cdot \cos ($ angle $)) /$

np.sin (angle)

$\mathrm{y} 1=($ dist $-\operatorname{cols} * \mathrm{np} \cdot \cos (\operatorname{angle})) / \mathrm{np} \cdot \sin ($ angle)

\# Create a plot of the results

plt.figure (figsize $=(10,9))$

plt.subplot $(2,2,1$, aspect=' equal')

plt.pcolormesh (medp90, cmap=plt.cm.gray)

plt.xlabel ('Distance [m]', fontsize=25)

plt.ylabel ('Distance [m]', fontsize=25) plt.title(' (a)

Filtered Data', fontsize=27)

plt.subplot $(2,2,(3,4))$ plt.imshow (np.log $(1+h)$, extent $=[n p \cdot r a d 2 \operatorname{deg}($ theta $[-1]), n p \cdot r a d 2 d e g($ theta $[0])$,

$d[-1], d[0]]$,

cmap=plt.cm.gray)\#, aspect=1/1.5) plt.title('(c) Hough

transform', fontsize=27) plt.xlabel ('Angles

[degrees]', fontsize=25) plt.ylabel ('Distance

[pixels]', fontsize=25)

plt. subplot $(2,2,2$, a spect=' equal' $)$

plt.pcolormesh (splitl, $\mathrm{cmap}=\mathrm{cm}$. terrain, vmin=28,

vmax=32) plt.plot ( $(0, \mathrm{cols}),(\mathrm{y} 0, \mathrm{y} 1), '-$

$r^{\prime}$, linewidth=3.5) plt.ylim([0,50]) 


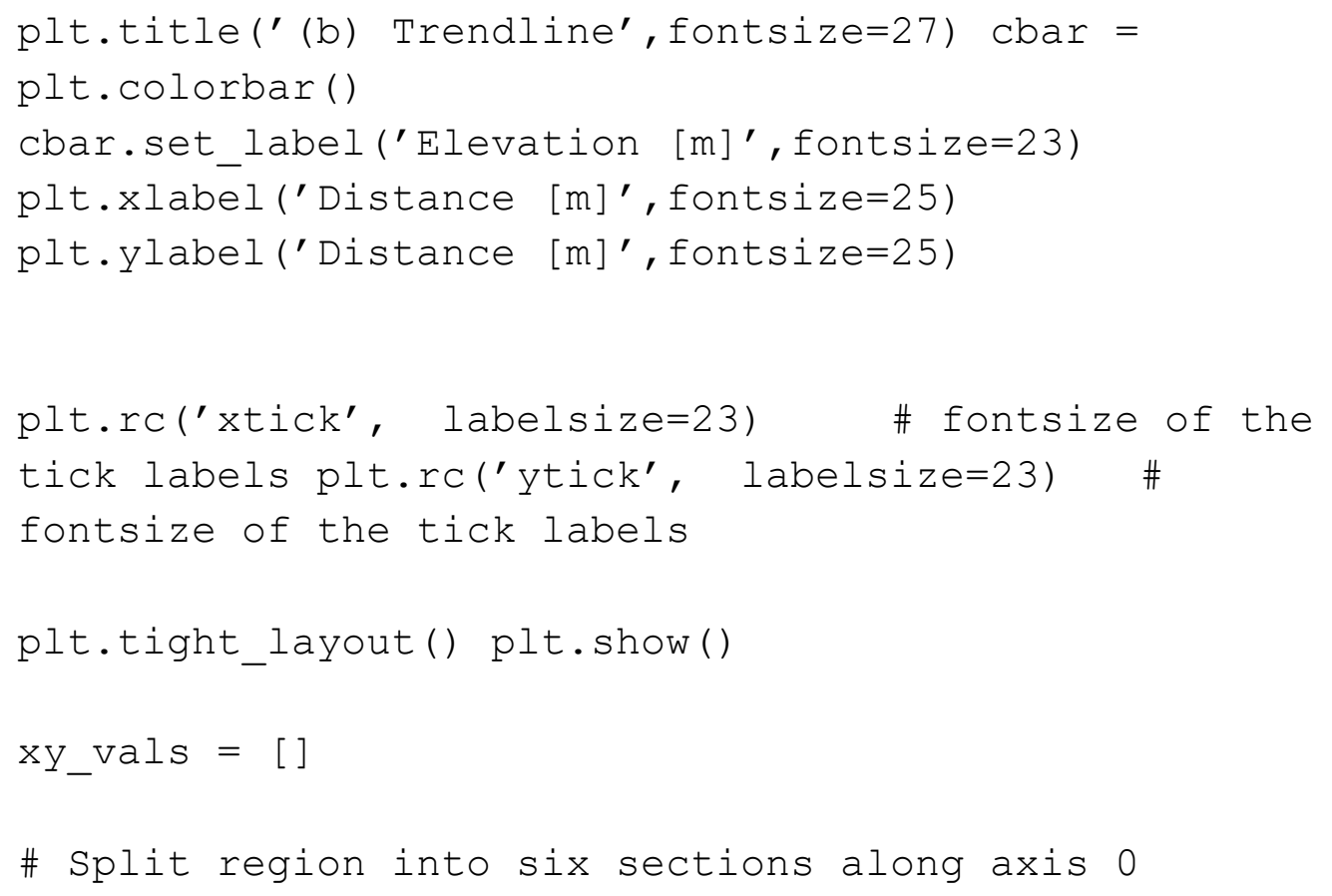


plt.suptitle('Ridge-Swale Region along the Mississippi

River', fontsize=30) plt.xlabel ('Pixels', fontsize=28)

plt.ylabel ('Pixels', fontsize=28)

for $x y$ in $x y \_v a l s:$

plt.plot (xy[0], xy[1],'-r', linewidth=3.5)

plt.locator_params (axis=' $x^{\prime}$, nbins=5) plt.show() 


\section{Unit Conversion Factors}

\begin{tabular}{|l|c|l|}
\hline Multiply & By & To Obtain \\
\hline degrees (angle) & 0.01745329 & radians \\
\hline feet & 0.3048 & meters \\
\hline miles (U.S. statute) & $1,609.347$ & meters \\
\hline square miles & $2.589998 \mathrm{E}+06$ & square meters \\
\hline
\end{tabular}




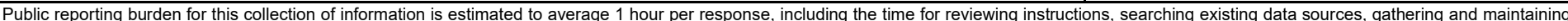

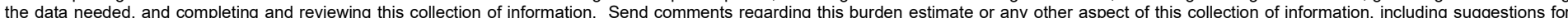

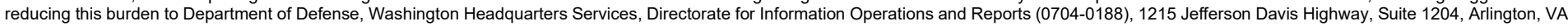

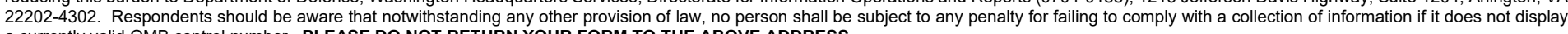
a currently valid OMB control number. PLEASE DO NOT RETURN YOUR FORM TO THE ABOVE ADDRESS.
1. REPORT DATE (DD-MM-YYYY) 2. REPORT TYPE
3. DATES COVERED (From - To) April 2021

\section{TITLE AND SUBTITLE} Final

Automated Characterization of Ridge-Swale Patterns Along the Mississippi River 5a. CONTRACT NUMBER

Automated Characterization of Ridge-Swale Patterns Along the Mississippi River

\section{5b. GRANT NUMBER}

5c. PROGRAM ELEMENT NUMBER

\section{AUTHOR(S)}

5d. PROJECT NUMBER

Alicia D. Downard, Stephen N. Semmens, and Bryant A. Robbins

5e. TASK NUMBER

5f. WORK UNIT NUMBER

\section{PERFORMING ORGANIZATION NAME(S) AND ADDRESS(ES)}

Geotechnical and Structures Laboratory

U.S. Army Engineer Research and Development Center

3909 Halls Ferry Road

Vicksburg, MS 39180-6199

\section{SPONSORING / MONITORING AGENCY NAME(S) AND ADDRESS(ES)}

U.S. Army Corps of Engineers

Washington, DC 20314-1000

8. PERFORMING ORGANIZATION REPORT
NUMBER

NUMBER

ERDC/GSL TR-21-13

10. SPONSOR/MONITOR'S ACRONYM(S)

11. SPONSOR/MONITOR'S REPORT NUMBER(S)

\section{DISTRIBUTION / AVAILABILITY STATEMENT}

Approved for public release; distribution is unlimited.

\section{SUPPLEMENTARY NOTES}

Flood \& Coastal Civil Works Direct Program, Remote Monitoring \& Sensing; Critical Infrastructure Protection and Resilience Program, Office of Homeland Security (Funding Account Code U4375173, AMSCO Code 031398)

\section{ABSTRACT}

The orientation of constructed levee embankments relative to alluvial swales is a useful measure for identifying regions susceptible to backward erosion piping (BEP). This research was conducted to create an automated, efficient process to classify patterns and orientations of swales within the Lower Mississippi Valley (LMV) to support levee risk assessments. Two machine learning algorithms are used to train the classification models: a convolutional neural network and a U-net. The resulting workflow can identify linear topographic features but is unable to reliably differentiate swales from other features, such as the levee structure and riverbanks. Further tuning of training data or manual identification of regions of interest could yield significantly better results. The workflow also provides an orientation to each linear feature to support subsequent analyses of position relative to levee alignments. While the individual models fall short of immediate applicability, the procedure provides a feasible, automated scheme to assist in swale classification and characterization within mature alluvial valley systems similar to LMV.

\begin{tabular}{|c|c|c|c|c|c|}
\hline \multicolumn{2}{|c|}{ 15. SUBJECT TERMS } & \multicolumn{2}{|c|}{ Artificial intelligence } & \multicolumn{2}{|c|}{ Alluvial plains } \\
\hline \multicolumn{2}{|c|}{ Geomorphology } & \multicolumn{2}{|c|}{ Backward erosion piping } & \multicolumn{2}{|c|}{ Alluvium } \\
\hline \multicolumn{2}{|c|}{ Ridge and swale topography } & \multicolumn{2}{|l|}{ Sand boils } & \multicolumn{2}{|c|}{ Alluvial fans } \\
\hline \multicolumn{2}{|c|}{ Mississippi River } & \multicolumn{2}{|l|}{ Levees_Erosion } & \multicolumn{2}{|c|}{ Digital elevation models } \\
\hline \multicolumn{2}{|l|}{ Flood control } & \multicolumn{2}{|l|}{ Geology } & \multicolumn{2}{|c|}{ Machine learning } \\
\hline \multicolumn{3}{|c|}{ 16. SECURITY CLASSIFICATION OF: } & \multirow[t]{3}{*}{$\begin{array}{l}\text { 17. LIMITATION } \\
\text { OF ABSTRACT }\end{array}$} & \multirow[t]{3}{*}{$\begin{array}{l}\text { 18. NUMBER } \\
\text { OF PAGES }\end{array}$} & $\begin{array}{l}\text { 19a. NAME OF RESPONSIBLE } \\
\text { PERSON }\end{array}$ \\
\hline a. REPORT & b. ABSTRACT & c. THIS PAGE & & & 19b. TELEPHONE NUMBER (include \\
\hline Unclassified & Unclassified & Unclassified & & & \\
\hline
\end{tabular}


